\title{
Pain as the neglected adverse event
}

Previously published at www.cmaj.ca

$\mathrm{T}$ he Institute of Medicine's groundbreaking 1999 report To Err is Human: Building a Safer Health System transformed attitudes and behaviour toward patient safety, but ignored one essential aspect. Many patients suffer from a specific adverse event on a daily basis: pain. It is never reported as an adverse event and corrective action is often not taken. Health care professionals are routinely aware of it, yet dismiss it as an unavoidable consequence of medical care.

Medically caused pain (postoperative and procedural) is our dirty little secret that we pretend is not an adverse event.

According to the American Society for Healthcare Risk Management, an adverse event is "a negative or bad result stemming from a diagnostic test, medical treatment or surgical intervention."1 Almost all patients believe pain is a major negative consequence of surgery or of diagnostic procedures ${ }^{2}$ and pain clearly results from surgical and procedural interventions (which cause nociceptive, inflammatory and/or neuropathic pain). If we accept that medically caused pain satisfies the definition of an adverse event, then why has it been left off the patient safety agenda?

Perhaps we believe that pain is not 'negative enough' to be an adverse event or we accept that pain cannot be minimized. We know that pain has negative impacts and while we acknowledge that not all pain can be avoided (indeed most patients believe that some pain can be tolerated without treatment $^{3}$ ) we do know that postoperative pain can be minimized and procedural pain can be prevented or reduced. Pain as an adverse event may be a controversial concept to health care providers or administrators, but it is not at all controversial from the patient's perspective.

Altering our conceptualization of pain from an unavoidable consequence of skin-breaking procedures to a preventable adverse event could have farreaching implications for clinical prac-

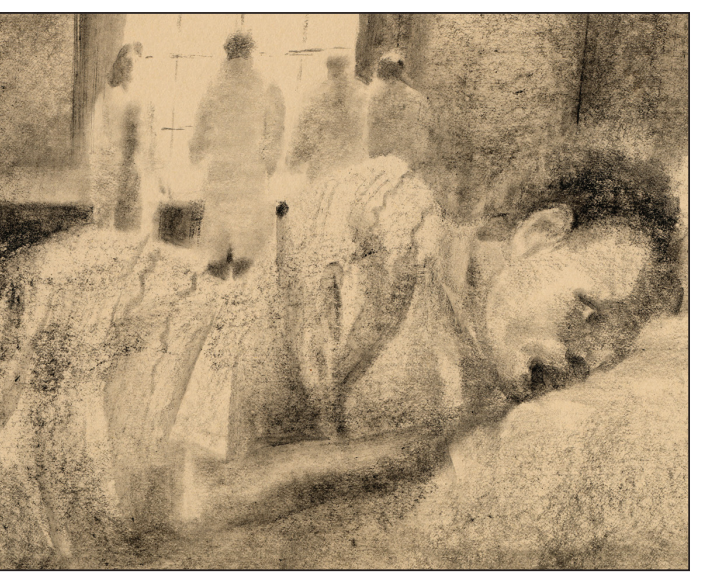

Eliminating medically caused pain is a goal that the health system must embrace. Best practice standards have been developed, like those by the American Society of Anesthesiologists Task Force on Acute Pain Management, the Association of Paediatric Anaesthetists in the United Kingdom, the Canadian Paediatric Society, the American Academy of Pediatrics and other groups.

Further research on pharmacologic and non-pharmacologic treatments is underway. Despite this work, barriers to eliminating postoperative and procedural pain have not been overcome. Treating excessive pain as an adverse event will ensure that best prac-

tice. Using an adverse event framework, standard care would be defined as minimal postoperative or procedural pain and documentation would be required when this standard was not met. In this way, protocols would highlight contexts in which pain treatment failed. Adverse event reporting would include information on the type of failure (e.g., communication, clinical performance), the characteristics of the environment, the individuals involved and the efforts taken to mitigate the effects of pain. Root cause analysis could be used to identify patient, provider, treatment and, perhaps most importantly, system-level variables that contribute to postoperative pain.

This process could lead to novel integrated approaches to pain management, including modifications to pharmacological management and integration of non-pharmacologic and rehabilitative interventions. Most significantly, considering pain to be an adverse event would place accountability for management at a systemic rather than an individual level. Adverse event reporting and analysis would result in an increased burden for health care professionals, but this would be relieved by systems of care that use best practices in pain management. tices are followed and obstacles to adequate management are identified and addressed, and recurrent unnecessary cruelty to patients will be eliminated.

\section{Jill MacLaren Chorney PhD}

Assistant professor of anesthesiology

Dalhousie University

Patrick McGrath OC PhD

Vice-president, research

IWK Health Centre

\section{G. Allen Finley MD}

Medical director, pediatric pain management

IWK Health Centre

Halifax, NS

\section{REFERENCES}

1. American Society for Healthcare Risk Management. Perspective on disclosure of unanticipated outcome information. Chicago (IL): The Society; 2001.

2. Apfelbaum JL, Chen C, Mehta SS, et al. Postoperative pain experience: results from a national survey suggest postoperative pain continues to be undermanaged. Anesth Analg 2003;97:534-40.

3. Gauthier JC, Finley GA, McGrath PJ. Children's self report of postoperative pain intensity and treatment threshold: determining the adequacy of medication. Clin J Pain 1998;14:116-120.

Have you got an opinion about this article? Post your views at www.cmaj.ca. Potential Salon contributors are welcome to send a query to salon@ cmaj.ca. 